\title{
A propósito del artículo: “Conocimiento y uso de método anticonceptivo de emergencia en estudiantes universitarios, Pereira, Colombia"
}

Sr. Editor, si bien se reconoce al Método Anticonceptivo de Emergencia (MAE) como un pilar fundamental en el control de la natalidad, este acarrea efectos adversos que podrían resultar nocivos para la salud de la mujer(1). De ahí la importancia del análisis exhaustivo realizado por el autor, quien pretende valorar hasta qué punto es conveniente restringir su adquisición (cabe recalcar que dichos efectos secundarios, además, varían de mujer a mujer, siendo el levonorgestrel la base fundamental de esta línea de fármacos).

En este contexto, asumiendo que el MAE se emplee de manera indiscriminada y ajena a las recomendaciones de la Organización Mundial de la Salud (OMS), los efectos secundarios conllevarían a: irregularidades en el ciclo menstrual (adelantándolo o postergándolo), embarazos ectópicos, adelgazamiento de las paredes uterinas, alteraciones en el proceso ovulatorio (lo que con el tiempo podría conducir a infertilidad), un posible embarazo (si el endometrio está formado, no se impide la fecundación) y alteraciones uterinas (hemorragias que impiden la implantación, sangrados intermenstruales, miomas)(2). Así, un estudio en México que describe los efectos adversos a la hora de emplear el MAE, expuso que, de las 330 adolescentes evaluadas, 189 reportaron: migraña $(12,2 \%)$, alopecia $(8,5 \%)$, acné $(15,3 \%)$, cambios menstruales $(34,4 \%)$, sangrado abundante $(16,4 \%)$, edema $(3,2 \%)$, insuficiencia venosa $(5,3 \%)$, hirsutismo $(4,8 \%)(3)$. De la misma manera, un estudio realizado en el Hospital de Paz Internacional para la Maternidad y Salud Infantil, Shanghái, tras hacer un seguimiento abocado a la evaluación del riesgo fetal con levonorgestrel, detectó riñones poliquísticos congénitos en las segundas visitas de seguimiento del embarazo (24 semanas de gestación); se evidenciaron, además, dos malformaciones fetales correspondientes a tumor sacro coccígeo y acondroplasia con enanismo de extremidades cortas; sin embargo, no se constata que dichas malformaciones sean debidas al empleo de levonogestrel(4).

Al margen de la terapéutica, nos vemos en la necesidad de hacer hincapié en su uso inapropiado como método anticonceptivo de uso regular, debido al incremento de la probabilidad de fracaso; consideramos pues, su uso exclusivo en mujeres fértiles expuestas a relaciones sexuales sin protección, ya sea debida al descuido propio, a la falla del método anticonceptivo de rutina, o por haber sido sujetas a abuso sexual. Finalmente, a pesar de la sugerencia del empleo del MAE a no más de dos tomas al año(5), un estudio realizado en México concluye que las adolescentes consumen de una a treinta y seis píldoras de emergencia en el lapso de un año(6); sin embargo, esta data no necesariamente explica la realidad que se da en otros países, por lo que se requiere de estudios que verifiquen esta condición de sobreuso. Se sugiere la implementación de programas informativos sobre MAE y otros métodos anticonceptivos de uso regular; además, dichos temarios y planificación, en general, deberán ser delegados por personal sanitario capacitado, a fin de garantizar una prevención segura, responsable y reducir al máximo las repercusiones que implicaría su uso inadecuado.

\section{Elizabeth Núñez-Quispe ${ }^{1 *}$} Samanta Picón 1

1 Escuela de Medicina Humana, Facultad de Ciencias de la Salud, Universidad Privada Norbert Wiener. Lima, Perú

Conflictos de interés: No declaran. 


\title{
Referencias
}

1. Acevedo-Osorio GO, Ramírez-García N, Cardona-Osorio JD, Oliveros-Ruiz CA. Conocimiento y uso de método anticonceptivo de emergencia en estudiantes universitarios, Pereira, Colombia. Univ. Salud. 2019;21(2):159-165.

2. Contreras-Mandujano JC, Reyes-de la Cruz C, Sarmiento-Arena Z, Murillo-Palma AL, Osorio-Alejo DR. Conocimiento y uso de la píldora de emergencia en jóvenes universitarias de Tabasco. Revista Horizonte Sanitario. 2018;17(3):227-234.

3. Pinzón-Martínez M, López-Wilches F. Percepción sobre las reacciones adversas del método anticoncepción de emergencia, en la población femenina fértil en el periodo de julio 2016 a junio 2017 en la zona 10 de Engativa del noroccidente de Bogotá DC. Bogotá DC: Universidad de Ciencias Aplicadas Y Ambientales; 2017.

4. Lin-Zhang, Junling-Chen, Yasun-Wang, Fangming-Ren, Wen-Yu, Linan-Cheng. Pregnancy outcome after levonorgestrelonly emergency contraception failure. A prospective cohort study. Human Reproduction. 2009;24(7):1605-11.

5. Blandón-García MC, Sandino-García, AC. Conocimiento, actitudes y prácticas sobre el uso de levonorgestrel como método anticonceptivo de emergencia de las estudiantes de la carrera de química-farmacéutica, departamento de química, recinto universitario "Rubén Darío" UNAN-Managua del 1 de octubre al 31 diciembre del 2014. Managua: Universidad Nacional de Nicaragua; 2015.

6. Villegas-Díaz JS, Martínez-Silva LA, Santiago-Pineda MD, Quinteros-Avendaño RS. Riesgos y daños a la salud de las adolescentes por el uso de la pastilla de emergencia. CuidArte "El arte del cuidado". 2012;1(1):34-42.

\section{Respuesta a Carta al editor}

\section{Métodos anticonceptivos de emergencia: Una prioridad de educación}

\author{
Germán Oved Acevedo-Osorio ${ }^{1}$ orcid.org/0000-0002-1499-2579 \\ Angélica María Blanco-Vanegas ${ }^{2}$ orcid.org/0000-0001-7021-1944 \\ Nicolás Ramírez-García1 ${ }^{1}$ orcid.org/0000-0001-5248-1561 \\ Julián David Cardona-Osorio ${ }^{1}$ orcid.org/0000-0003-1240-7409 \\ Christian Alexander Oliveros-Ruiz ${ }^{1}$ orcid.org/0000-0003-0622-9642
}

1 Grupo de Investigación Los Asépticos, Fundación Universitaria del Área Andina - Seccional Pereira. Pereira, Colombia

2 Grupo de Investigación ZIPATEFI, Fundación Universitaria del Área Andina - Seccional Pereira. Pereira, Colombia

Los métodos anticonceptivos hormonales orales de emergencia han evolucionado desde el primer uso de altas dosis de estrógeno en la década de $1960^{(1)}$ y el levonorgestrel es considerado en la actualidad como la base fundamental de esta línea de fármacos como se relaciona en la carta al editor titulada; A propósito del artículo: "Conocimiento y uso de método anticonceptivo de emergencia en estudiantes universitarios, Pereira, Colombia".

A diferencia de la píldora anticonceptiva utilizada regularmente, que tiene una mayor aceptación social, la anticoncepción de emergencia suscita mucha controversia. Se exploran dos perspectivas analíticas: la primera es la estrategia que señala los peligros y posibles riesgos para la salud que representaría este anticonceptivo, con miras a regular los cuerpos femeninos; especialmente jóvenes y pobres, donde se teme la reproducción. El segundo analiza la agencia femenina que muestra el uso de la anticoncepción de emergencia, una perspectiva que puede estar perjudicando las jerarquías morales y sociales, de clase y de género.

El uso y distribución inadecuado de levonorgestrel, sin educación apropiada a la comunidad, genera prejuicios y estigmas frente a su consumo. Para la población, el poder de acción de las hormonas en el cuerpo puede ser devastador, cuando se usan en exceso, causando un desequilibrio orgánico. A pesar de ser 
un método altamente efectivo, el uso prolongado y/o irrazonable del levonorgestrel puede causar grandes daños a la salud de las mujeres, con énfasis en el cáncer de mama y de cuello uterino, así como la disminución de la eficacia terapéutica con posible embarazo no deseado e infertilidad. Además, este método no proporciona protección para infecciones de transmisión sexual. Las percepciones sobre los riesgos de la anticoncepción de emergencia están relacionadas con la creencia de que se trata de un medicamento "más fuerte" y "peligroso", de ahí la mención del término "bomba hormonal" como lo menciona el artículo de Brandão, et al.(2).

En relación a las campañas de prevención de embarazos no deseados, se hace especial énfasis en la difusión y educación frente al uso y control de dicho régimen de anticonceptivos, evidenciable en un reciente estudio, donde los resultados mostraron que la tasa de embarazo después del tratamiento con levonorgestrel en mujeres en un entorno clínico real, fue baja y similar a la informada en estudios anteriores ${ }^{(3)}$.

Según el Ministerio de Salud colombiano, la eficacia comparativa entre los dos regímenes hormonales; el levonorgestrel evitó el 85\% de los embarazos que hubieran ocurrido sin antinconcepción de emergencia, mientras que el Yuzpe sólo el 57\% según datos ofrecidos por esta organización(4). La información sobre métodos anticonceptivos regulares y anticoncepción de emergencia con levonorgestrel debe difundirse mejor y con estrategias focalizadas entre las mujeres en edad de procrear como se planteó en nuestro estudio inicial(5).

Conflictos de interés: Ninguno.

\section{Referencias}

1. Benagiano G, Carrara S, Filippi V. Safety, efficacy and patient satisfaction with continuous daily administration of levonorgestrel/ethinylestradiol oral contraceptives. Patient Prefer Adherence. 2009;3:131-43.

2. Brandão ER, Cabral CdaS, Ventura M, Paiva SP, Bastos LL, Oliveira NVBV, et al. "Bomba hormonal": os riscos da contracepção de emergência na perspectiva dos balconistas de farmácias no Rio de Janeiro, Brasil. Cad Saúde Pública, Rio Janeiro. 2016;32(9):1-11. doi: 10.1590/0102-311X00136615

3. Sakurai S. Pregnancy rate after emergency contraception with single-dose oral levonorgestrel in Japanese women. J Obstet Gynaecol Res. 2019;45(9):189-98. doi: 10.1111/jog.14049

4. Ministerio de Salud y Protección Social. Anticoncepción de emergencia. Bogotá DC: MinSalud; 2019.

5. Acevedo-Osorio GO, Ramírez-García N, Cardona-Osorio JD, Oliveros-Ruiz C. Conocimiento y uso de método anticonceptivo de emergencia en estudiantes universitarios, Pereira, Colombia. Univ. Salud. 2019;21(2):159-65. 